\title{
Technique for the safe placement of a biodegradable stent into the common bile duct of rabbits
}

\author{
YIGANG CHEN $^{1 *}$, JUN YAN $^{1,2, *}$, ZHIGANG WANG $^{2}$, SONG YU $^{2}$, ZIMING YUAN $^{1}$, \\ XIAOHU WANG ${ }^{2}$, XIAONONG ZHANG ${ }^{3}$ and QI ZHENG ${ }^{1,2}$ \\ ${ }^{1}$ Shanghai Jiaotong University School of Medicine, Shanghai 200025; ${ }^{2}$ Department of General Surgery, \\ Sixth People's Hospital, Shanghai Jiao Tong University School of Medicine, Shanghai 200233; \\ ${ }^{3}$ State Key Laboratory of Metal Matrix Composites, School of Materials Science and Engineering, \\ Shanghai Jiao Tong University, Shanghai 200240, P.R. China
}

Received April 10, 2013; Accepted July 25, 2013

DOI: $10.3892 /$ etm.2013.1276

\begin{abstract}
Biodegradable common bile duct (CBD) stents are in high clinical demand. Animal experiments concerning the surgical placement of biliary stents made of new materials are being performed more frequently than ever before. However, these animal experiments only use large animals. In this study, a central venous catheterization set was used as a modified stent introducer system in rabbits. A biodegradable $\mathrm{Mg}-6 \mathrm{Zn}$ alloy CBD stent was passed through the duodenal papilla using this stent introducer system. Computed tomography (CT) scanning of the CBD stent in vivo and levels of serum lipase (LPS) were investigated. Twelve rabbits underwent CBD stent insertion and one animal died due to an anesthetic accident. After 3 weeks, when the remaining 11 rabbits were sacrificed, no jaundice or bile leakage was observed. CT scanning of the 11 rabbits suggested that the biodegradable Mg-6Zn stent was successfully placed into the CBD. When the preoperative and postoperative levels of LPS were compared, no statistically significant differences were observed. This new method appears to be feasible and safe for the placement of stents into the CBDs of small animals. This new method can increase the animal number of CBD stent experiment, and improve the quality of experiments.
\end{abstract}

\section{Introduction}

Diseases of the common bile duct (CBD) represent a significant danger to the patient, since they may lead to obstructive

Correspondence to: Dr Qi Zheng, Department of General Surgery, Sixth People's Hospital, Shanghai Jiao Tong University School of Medicine, 600 Yishan Road, Shanghai 200233, P.R. China

E-mail: shanghaizhengqi@163.com

*Contributed equally

Abbreviations: CBD, common bile duct

Key words: biodegradable stent, animal experiment, common bile duct jaundice, biliary colic, cholangitis or pancreatitis $(1,2)$. Traditionally, surgeons explore the $\mathrm{CBD}$ and then conduct T-tube drainage following the removal of CBD stones or lesions. It is believed that a T-tube is necessary since it allows spasm or edema of sphincter to settle following the trauma of the exploration. Despite this potential advantage, T-tube-associated complications, including CBD obstruction and bile leakage, often occur after CBD surgery (3-5). An indwelling T-tube requires prescription by a doctor, continuous management and the restriction of patient activity. At the end of treatment, the process of T-tube removal may cause pain and risk to the patient (6-8). The avoidance of complications of the T-tube and the need to support the bile duct are the major challenges faced by surgeons and researchers.

Since biodegradable implant materials in the human body may be gradually dissolved and absorbed, secondary surgery to remove the implants is not required (9). To date, a great number of studies have reported on biodegradable CBD stents in animal experiments (10-12). However, there are certain limitations for the animal experiments, since they are often conducted in large animals such as pigs or dogs (usually with a small sample size of 4-7 dogs or pigs). Large animals have a relatively large CBD which is convenient for the CBD surgery. However, large animals such as dogs and pigs have two disadvantages. Firstly, they are expensive, which limits the number of experimental animals that may be used. Secondly, there are few antibodies against pigs or dogs, which add extra difficulties for molecular experiments in the CBD.

The current study describes a new technique designed for the placement of biodegradable stents into the CBDs of rabbits. The purpose of this study was to provide a safe CBD surgical method in order to enable the sample size of the animals to be expanded and to increase the credibility of experiments concerning biodegradable CBD stents in animals.

\section{Materials and methods}

Biodegradable CBD stent and stent introducer system. The biodegradable stents made of $\mathrm{Mg}-6 \mathrm{Zn}$ alloy, donated by Shanghai Origin Material and Medical Technology Co. Ltd. (Shanghai, China), were made with high purity Mg (99.99\%) 
Table I. Chemical composition of Mg-6Zn alloy.

\begin{tabular}{lcccccccr}
\hline & \multicolumn{7}{c}{ Chemical composition (weight \%) } \\
\cline { 2 - 9 } Material & $\mathrm{Fe}$ & $\mathrm{Si}$ & $\mathrm{Ni}$ & $\mathrm{Cu}$ & $\mathrm{Al}$ & $\mathrm{Mn}$ & $\mathrm{Zn}$ & $\mathrm{Mg}$ \\
\hline Mg-6Zn & 0.0038 & 0.0016 & 0.0005 & 0.0005 & 0.0085 & 0.0004 & 5.6210 & 94.3637 \\
\hline
\end{tabular}

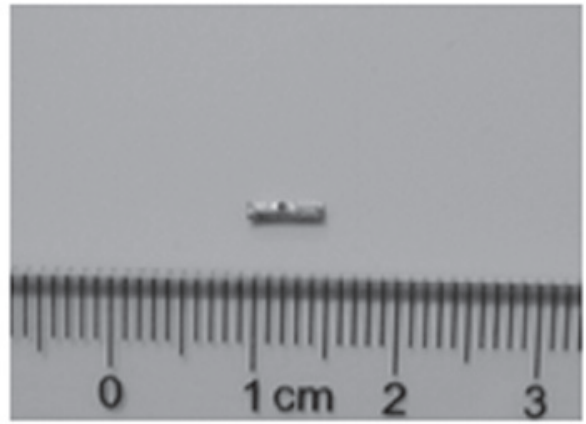

Figure 1. A biodegradable biliary stent made of Mg-6Zn alloy, $1 \mathrm{~mm}$ in diameter and $5 \mathrm{~mm}$ in length. The stent is U-shaped and has a small hole drilled in it.

and high purity zinc (Zn; 99.999\%) under a clean process. The chemical composition of the Mg-6Zn alloy is shown in Table I. Materials were prepared as previously described (13). The biodegradable biliary stents were U-shaped and possessed a luminal diameter of $1.0 \mathrm{~mm}$ and a length of $5 \mathrm{~mm}$ (Fig. 1). A small hole was drilled in the middle of the stent to enable the stent to be sutured to the CBD wall. The system used in endoscopic retrograde cholangiopancreatography (ERCP) prompted the use a central venous catheterization set (REF CS-24301-E; Arrow International, Inc., Reading, PA, USA) as a special stent introducer system (Fig. 2). The parts used included a plastic jacket tube and a metal guide wire, which formed the CBD introducer system.

Animal model and study design. The animal experiment was conducted according to the Guidance Suggestions for the Care and Use of Laboratory Animals (issued by the Ministry of Science and Technology of the People's Republic of China), and was approved by the Ethics Committee of the Sixth People's Hospital of Shanghai Jiao Tong University (Shanghai, China). The animals were supplied by the Sino-British Sippr/BK Lab Animal Ltd., Co. [license no. SCXK(hu)2008-0016; Shanghai, China]. Twelve adult New Zealand rabbits with a mean body weight of $2.5 \pm 0.5 \mathrm{~kg}$ were used in the experiments.

Rabbits were placed under general anesthesia by the intravenous administration of sodium pentobarbital at a dose of $30 \mathrm{mg} / \mathrm{kg}$ body weight. All surgical procedures were carried out under sterile conditions. Through a midline laparotomy, the $\mathrm{CBD}$ was exposed and dissected along the $\mathrm{CBD}$ to $10 \mathrm{~mm}$ from the duodenal wall. The mean diameter of the CBD was $1.5 \mathrm{~mm}$ and the mean length was $12 \mathrm{~mm}$ in 10 rabbits. A ligature was loosely placed on the CBD as a marker of the location of desired stent placement. A $15-\mathrm{mm}$ longitudinal incision was made in the duodenum, $5 \mathrm{~mm}$ away from the
A

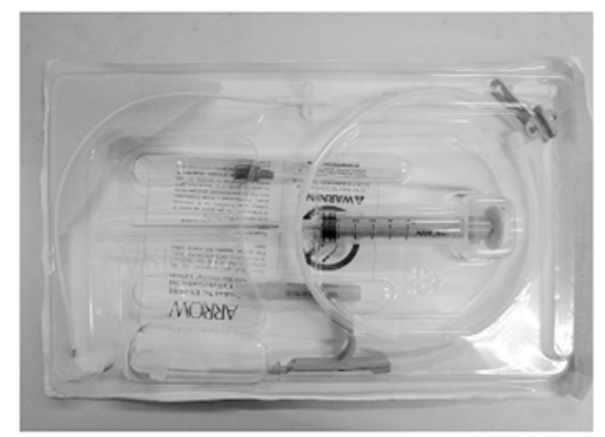

B

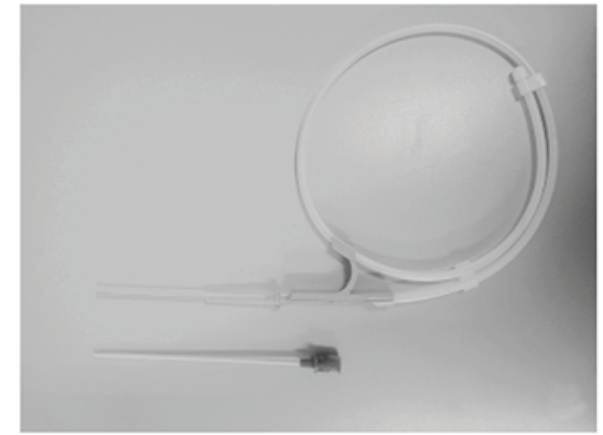

C

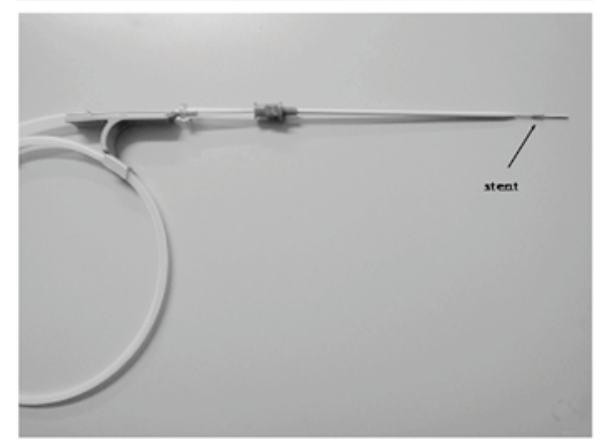

Figure 2. A central venous catheterization set (REF CS-24301-E; Arrow International, Inc.) was used as a special stent introducer system. (A) The central venous catheterization set; (B) a plastic jacket tube and a metal guide wire. (C) The stent was mounted onto the stent introducer system.

duodenal papilla. The stents were mounted onto the special stent introducer system. When the metal guide wire arrived at the ligature marker on the CBD, the plastic jacket tube was pushed back. Then, the stent was advanced along the duodenal papilla and the metal guide wire was withdrawn. As the CBDs of rabbits are translucent, it was possible to view the stent in the CBD under direct vision and to suture the stent to the CBD wall through the small hole on the stent. The plastic jacket tube was then withdrawn, and the duodenum and abdomen was closed (Fig. 3).

Computed tomography (CT) scanning of the CBD stent in vivo was conducted after the surgical procedures immediately and postoperatively for 1-3 weeks. To investigate changes 

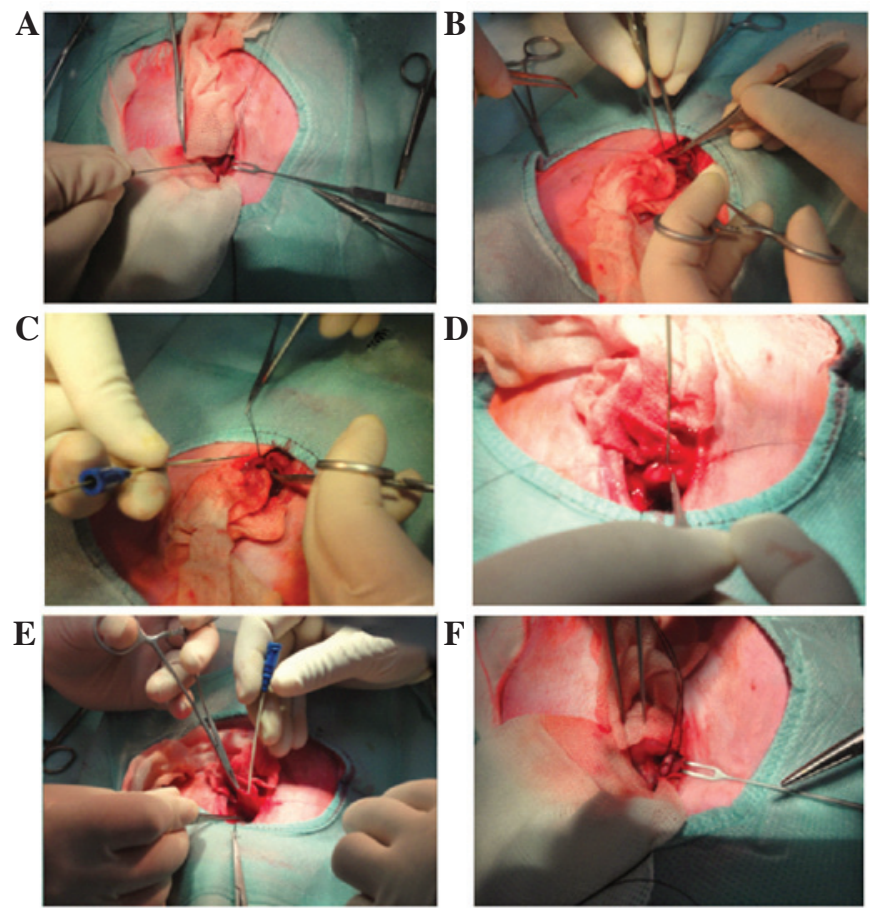

Figure 3. Process for placing an advanced stent into the rabbit common bile duct (CBD). (A) The CBD was identified and ligatured as a marker; (B) a $15-\mathrm{mm}$ longitudinal incision was cut into the duodenum, $5 \mathrm{~mm}$ away from the duodenal papilla; (C) the stent introducer system was assembled and prepared to place the stent; (D) a metal guide wire was placed into the CBD, the plastic jacket tube was pushed down and a stent was inserted into the CBD; (E) the guide wire and plastic jacket tube were gradually withdrawn; (F) the stent was sutured to the $\mathrm{CBD}$ inner wall.
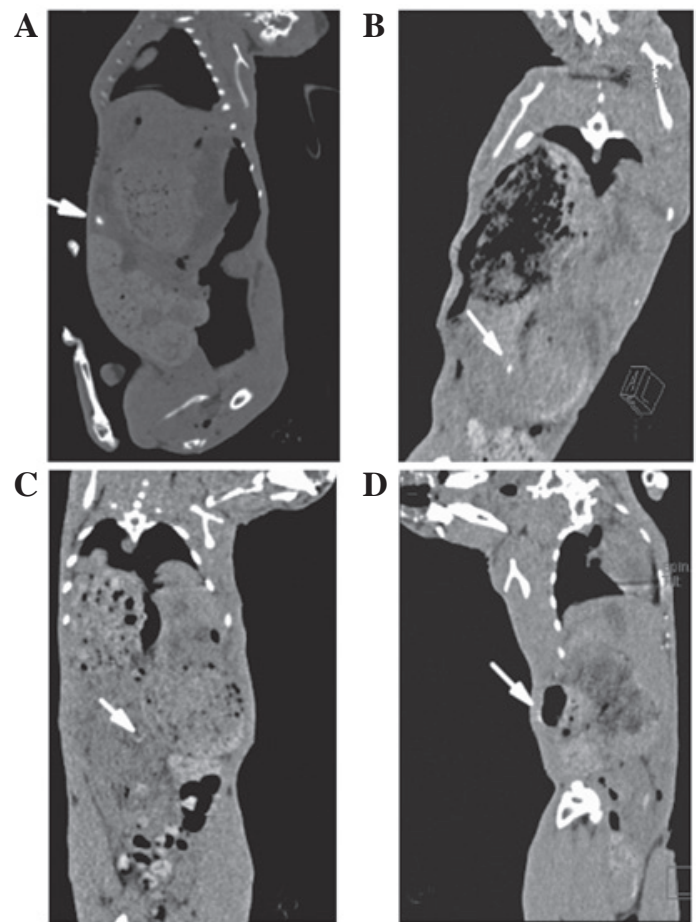

Figure 4. Computed tomography scan on (A) the day of surgery and (B) the 1st, (C) 2nd and (D) 3rd week post-implantation. The stents degraded gradually.

in the pancreatic function of the rabbits, the serum lipase (LPS) values of each rabbit were determined. In brief, a 1-ml

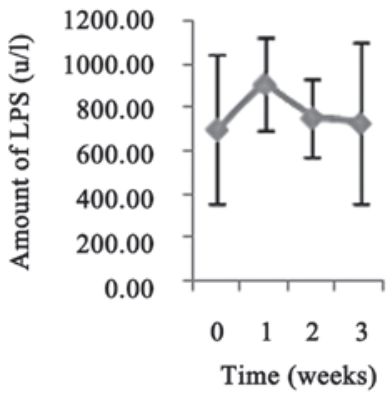

Figure 5. Serum lipase (LPS) levels of the rabbits prior to and following surgery.

blood sample was collected prior to the surgical procedures and postoperatively for 1-3 weeks from the ear vein of the rabbit using a vein puncture into a heparinized syringe. The samples were then immediately analyzed using an automatic blood biochemistry analyzer (Hitachi 7600-020; Hitachi High-Technologies, Tokyo, Japan; LPS kit was provided by Koch Industries, Inc., Wichita, KS, USA). Eleven rabbits were sacrificed at 3 weeks after surgery.

Statistical analysis. Statistical analysis was performed with SPSS 18.0 software package (SPSS Inc., Chicago, IL, USA). The experimental values were analyzed using the paired-samples t-test and expressed as the mean values \pm standard deviation (SD). One-way ANOVA analysis was calculated to determine differences between groups for each evaluated parameter at each time point. Non-parametric tests [ $\kappa$ independent samples tests (Kruskal-Wallis Test)] were calculated when equal variances were not assumed in one-way ANOVA. $\mathrm{P}<0.05$ was considered to indicate a statistically significant result.

\section{Results}

Twelve rabbits underwent CBD stent insertion using the stent introducer system. One animal died due to an anesthetic accident. The remaining 11 rabbits that were included in the final analysis grew well and their activities, diet and drinking were normal. When these 11 rabbits were sacrificed after 3 weeks, no jaundice or bile leakage was observed.

CT scanning for these 11 rabbits suggested that the biodegradable Mg-6Zn stent was successfully placed into the CBD. Since the stents degraded gradually, after 3 weeks, the stent was rarely identified (Fig. 4). Although the levels of LPS 1 week postoperatively were slightly higher than the preoperative levels, no statistically significant differences were observed (P>0.05; Fig. 5).

\section{Discussion}

Following CBD exploration, it is important to insert a T-tube in the CBD to provide a support and to maintain an open CBD (14). However, T-tube drainage is controversial since it appears to prolong the hospital stay and is associated with an increased cost of care (15). There are risks for T-tube placement, including increased morbidity or mortality secondary to biliary infection, migration of the tube causing bile duct obstruction, or bile leaks or peritonitis following the removal 
of the tube $(1,16,17)$. Therefore, there is a great clinical requirement for biodegradable CBD stents. Animal experiments for new surgical biliary stents made of novel materials are being conducted more frequently than ever before. However, in practice, it is difficult to perform a safe choledochotomy for small animals, such as rabbits.

In the present study, a biodegradable Mg-6Zn alloy CBD stent was inserted into the CBDs of rabbits via a central venous catheterization set. At present, there are several materials that may be used to create a biodegradable stent and in which it is easy to drill a hole in the surface. Since the CBD of rabbits is translucent, it is possible to observe these stents in the $\mathrm{CBD}$, and the hole in the middle of the stent under direct vision. The stent was fixed to the CBD wall by a suture through the hole. Due to the stent compression of the sutured wall of CBD, no bile leakage was observed following suture completion. Fixing the stent to the CBD wall has certain advantages. Firstly, it demonstrates whether a stent may induce histological changes where it contacts with the CBD. Secondly, a fixed CBD stent is required for study of further stent biodegradation behavior .

One main problem to be overcome is the requirement that the progression of the stent through the duodenal papilla should be completed within a short time, which may reduce the adverse effects on the duodenal papilla. In order to clearly explore the duodenal papilla and the relationship between the papilla and the direction of the CBD, a relatively large longitudinal incision $(15 \mathrm{~mm})$ was cut in the duodenum, $5 \mathrm{~mm}$ away from the duodenal papilla. The results of serum LPS assays showed that this method for CBD stent placement had no significant impact on the function of the pancreas.

In conclusion, although the advancement of the stent through the duodenal papilla produces extra risks, our methods appear to be feasible and safe for the placement of stents into the CBDs of small animals. This study increases the number of animals available for CBD studies and may improve the quality of experiments concerning CBD stents in animals.

\section{Acknowledgements}

This study was supported by the National Natural Science Foundation of China (no. 30901422), Shanghai Jiao Tong University Interdisciplinary (Biomedical Engineering)
Research Fund (no. YG2010MS45) and Shanghai Jiao Tong University School of Medical and Technology Fund (no. 09XJ21005).

\section{References}

1. Verbesey JE and Birkett DH: Common bile duct exploration for choledocholithiasis. Surg Clin North Am 88: 1315-1328, 2008.

2. Hungness ES and Soper NJ: Management of common bile duct stones. J Gastrointest Surg 10: 612-619, 2006

3. El-Geidie AA: Is the use of T-tube necessary after laparoscopic choledochotomy? J Gastrointest Surg 14: 844-848, 2010.

4. Leida Z, Ping B, Shuguang W and Yu H: A randomized comparison of primary closure and T-tube drainage of the common bile duct after laparoscopic choledochotomy. Surg Endosc 22: 1595-1600, 2008.

5. Ahmed I, Pradhan C, Beckingham IJ, Brooks AJ, Rowlands BJ and Lobo DN: Is a T-tube necessary after common bile duct exploration? World J Surg 32: 1485-1488, 2008.

6. Daldoul S, Moussi A and Zaouche A: T-tube drainage of the common bile duct choleperitoneum: etiology and management. J Visc Surg 149: e172-e178, 2012.

7. Lygidakis NJ: Hazards following T-tube removal after choledochotomy. Surg Gynecol Obstet 163: 153-155, 1986.

8. Horgan PG, Campbell AC, Gray GR and Gillespie G: Biliary leakage and peritonitis following removal of $\mathrm{T}$ tubes after bile duct exploration. Br J Surg 76: 1296-1297, 1989.

9. Song G: Control of biodegradation of biocompatable magnesium alloys. Corros Sci 49: 1696-1701, 2007.

10. Xu X, Liu T, Liu S, Zhang K, Shen Z, Li Y and Jing X: Feasibility of biodegradable PLGA common bile duct stents: an in vitro and in vivo study. J Mater Sci Mater Med 20: 1167-1173, 2009.

11. Zografakis JG, Jones BT, Ravichardran P, EvanchoChapman MM, et al: Endoluminal reconstruction of the canine common biliary duct. Curr Surg 60: 437-441, 2003.

12. Laukkarinen J, Nordback I, Mikkonen J, Kärkkäinen P and Sand J: A novel biodegradable biliary stent in the endoscopic treatment of cystic-duct leakage after cholecystectomy. Gastrointest Endosc 65: 1063-1068, 2007.

13. Zhang E, Yin D, Xu L, Yang L and Yang K: Microstructure, mechanical and corrosion properties and biocompatibility of $\mathrm{Mg}-\mathrm{Zn}-\mathrm{Mn}$ alloys for biomedical application. Mater Sci Eng C Mater Biol Appl 3: 987-993, 2009.

14. Wu JS and Soper NJ: Comparison of laparoscopic choledochotomy closure techniques. Surg Endosc 16: 1309-1313, 2002.

15. Seale AK and Ledet WP Jr: Primary common bile duct closure. Arch Surg 134: 22-24, 1999.

16. Isla AM, Griniatsos J and Wan A: A technique for safe placement of a biliary endoprosthesis after laparoscopic choledochotomy. J Laparoendosc Adv Surg Tech A 12: 207-211, 2002.

17. Wu X, Yang Y, Dong P, et al: Primary closure versus T-tube drainage in laparoscopic common bile duct exploration: a meta-analysis of randomized clinical trials. Langenbecks Arch Surg 397: 909-916, 2012. 